\title{
Multiprobe AFM Bio-Imaging: The Next Evolution in SPM
}

\author{
Rimma Dekhter ${ }^{1}$, Galina Fish ${ }^{1}$, Sophia Kokotov ${ }^{1}$, Michael Kokotov ${ }^{1}$, Hesham Taha $^{1}$, David Lewis ${ }^{1}$ \\ Aaron Lewis ${ }^{2}$ \\ ${ }^{1}$ Nanonics Imaging Ltd, Jerusalem, Israel \\ ${ }^{2}$ The Hebrew University of Jerusalem, Jerusalem, Israel
}

Atomic force microscopy (AFM) with tuning fork feedback is the best method of AFM imaging known today. This presentation will describe the operation of this feedback mechanism in liquid. This allows for live cell AFM and NSOM operation in physiological media with high Q factors and without severe damping effects or any optical or mechanical constraints or interference. The extension of this frequency modulation feedback mechanism to tuning fork based liquid operation allows for scanned probe microscopy (SPM) cellular imaging fully integrated with any optical microscope including upright, 4 pi or standard Raman microprobes. It also will be shown that water immersion objectives can now be used with SPM and that these new directions allow for the first time live cell bioimaging with NSOM in spite of the stiff cantilevers that are generally associated with NSOM probes. The advances reported in this presentation, along with additional innovations in probe and scanner developments, allow for the dream of multiprobe NSOM/SPM to be implemented in physiological media. The results of these efforts portend important advances in the application of SPM in structural and functional bioimaging. 